\title{
Whole exome sequencing identifies ATRX mutation as a key molecular determinant in lower-grade glioma
}

\author{
Kasthuri Kannan ${ }^{1,4}$, Akiko Inagaki ${ }^{2}$, Joachim Silber ${ }^{1,4}$, Daniel Gorovets ${ }^{1,4}$, Jianan \\ Zhang $^{1,4}$, Edward R. Kastenhuber ${ }^{1,4}$, Adriana Heguy ${ }^{4}$, John H. Petrini ${ }^{2}$, Timothy A. \\ Chan $^{3,4}$, and Jason T. Huse ${ }^{1,4}$ \\ 1 Department of Pathology, Memorial Sloan-Kettering Cancer Center, New York, NY, USA \\ 2 Department of Molecular Biology, Memorial Sloan-Kettering Cancer Center, New York, NY, USA \\ ${ }^{3}$ Department of Radiation Oncology, Memorial Sloan-Kettering Cancer Center, New York, NY, USA \\ ${ }^{4}$ Department of Human Oncology and Pathogenesis Program, Memorial Sloan-Kettering Cancer Center, New York, NY, USA \\ Correspondence to: Jason T. Huse, email: husej@mskcc.org \\ Keywords: glioma, astrocytoma, IDH, ATRX, whole-exome sequencing \\ Received: October 01, 2012, Accepted: October 09, 2012, Published: October 11, 2012
}

Copyright: @ Kannan et al. This is an open-access article distributed under the terms of the Creative Commons Attribution License, which permits unrestricted use, distribution, and reproduction in any medium, provided the original author and source are credited.

\section{ABSTRACT:}

The molecular foundations of lower-grade gliomas (LGGs)-astrocytoma, oligodendroglioma, and oligoastrocytoma-remain less well characterized than those of their fully malignant counterpart, glioblastoma. Mutations in isocitrate dehydrogenase 1 and 2 (IDH1/2) likely represent initiating pathogenic events. However, while IDH mutations appear to dramatically alter cellular epigenomic landscapes, definitive downstream transformative mechanisms have not been characterized. It remains likely, therefore, that additional genomic abnormalities collaborate with IDH mutation to drive oncogenesis in LGG. We performed whole exome sequencing in 4 LGGs, followed by focused resequencing in an additional 28, and found a high incidence of mutations in the ATRX gene (a thalassemia/mental retardation syndrome X-linked). ATRX forms a core component of a chromatin remodeling complex active in telomere biology. Mutations in ATRX have been identified in multiple tumor types and appear to cause alternative lengthening of telomeres (ALT), a presumed precursor to genomic instability. In our samples, ATRX mutation was entirely restricted to IDH-mutant tumors, closely correlated with TP53 mutation and astrocytic differentiation, and mutually exclusive with 1p/19q codeletion, the molecular hallmark of oligodendroglioma. Moreover, ATRX mutation was highly enriched in tumors of so-called early progenitor-like transcriptional subclass $(\sim 85 \%)$, which our prior work has linked to specific cells of origin in the forebrain subventricular zone. Finally, ATRX mutation correlated with ALT, providing a mechanistic link to genomic instability. In summary, our findings both identify ATRX mutation as a defining molecular determinant for a large subset of IDH-mutant gliomas and have direct implications on pathogenic mechanisms across the wide spectrum of LGGs.

\section{INTRODUCTION}

Diffuse gliomas represent a biologically heterogeneous group of primary brain tumors whose shared propensity to widely infiltrate surrounding brain parenchyma renders them incurable, even in the face of ionizing radiation and cytotoxic chemotherapy [1]. Recent comprehensive genomic profiling has greatly clarified the molecular foundations of glioblastoma (GBM, WHO grade IV) the most common and malignant diffuse glioma variant [2-6], and promoted the exploration of an array of therapeutic strategies [7, 8]. By contrast, the underlying pathogenic events driving lower-grade diffuse gliomas (LGGs, WHO grade II and III) are considerably less clear. 
Table 1: Validated somatic mutations from whole exome sequencing of 4 IDH-mutant LGGs.

\begin{tabular}{|l|l|l|l|}
\hline Gene & Transcript Accession & Tumor & Amino Acid Change \\
\hline & & G4 & p.R132H \\
IDH1 & NM_005896.2 & G5 & p.R132G \\
& & G18 & p.R132H \\
& & G6 & p.R132H \\
\hline & & G5 & p.K1001fs \\
ATRX & & G18 & p.R1426X \\
& & G6 6 .L639fs \\
\hline TP53 & NM_000489.3 & G5 & p.R273C \\
& & G6 & p.R273C \\
\hline MUC4 & NM_018406.6 & G5 & p.D1413N \\
\hline MYBBP1A & NM_001105538.1 & G4 & p.K277N \\
\hline OR2B6 & NM_012367.1 & G18 & p.M118V \\
\hline ABCC11 & NM_032583.3 & G18 & p.V668M \\
\hline GABRB3 & NM_000814.5 & G5 & p.P145L \\
\hline TNFRSF11B & NM_002546.3 & G18 & p.L331M \\
\hline CR1 & NM_000651.4 & G6 & p.V1927L \\
\hline KIAA1731 & NM_033395.1 & G5 & p.L272P \\
\hline HERC2 & NM_004667.5 & G5 & p.S2763C \\
\hline KIAA1211 & NM_020722.1 & G5 & p.A1105V \\
\hline EXT2 & NM_000401.3 & G5 & p.N131K \\
\hline SRA1 & NM_001035235.3 & G5 & p.V110delinsGL \\
\hline
\end{tabular}

Current convention dictates that LGGs be designated as either astrocytoma, oligodendroglioma, or mixed glioma (oligoastrocytoma), primarily on the basis of histopathological criteria [9]. Recently, point mutations in the isocitrate dehydrogenase genes IDH1 and $\mathrm{IDH} 2$ have been identified in $70-90 \%$ of LGGs spanning across all morphologic subtypes [10-12]. IDH mutations induce a neomorphic enzymatic activity that preferentially generates the oncometabolite $\mathrm{R}(-)$-2-hydroxyglutarate at the expense of the normal TCA cycle component $\alpha$-ketoglutarate $[13,14]$. And while this process appears to cause widespread disruptions in cellular physiology, particularly with respect to the epigenome $[15,16]$, the precise mechanisms by which IDH mutation promotes tumorigenesis remain to be elucidated. In this setting, it is highly likely that additional molecular aberrations conspire with IDH mutation to induce tumorigenesis.

It has been known for some time that coordinated loss of chromosomes $1 \mathrm{p}$ and $19 \mathrm{q}(1 \mathrm{p} / 19 \mathrm{q}$ codeletion) designates a prognostically favorable LGG subgroup comprised primarily of oligodendrogliomas and, to a lesser extent, oligoastrocytomas, while largely excluding astrocytomas [17-19]. More recent work has demonstrated an almost invariable association between $1 \mathrm{p} / 19 \mathrm{q}$ codeletion and IDH mutation, implying that both abnormalities are required for transformation in tumors of fundamentally oligodendroglial lineage [11]. By contrast, the molecular landscape of LGGs harboring intact $1 \mathrm{p} / 19 \mathrm{q}$ - predominantly astrocytomas - appears less uniform, with mutations in TP53 representing their single most prevalent genomic anomaly apart from IDH mutation $[11,20]$. To gain a better understanding of this heterogeneity, we recently employed global transcriptional analysis to establish three molecularly and clinically distinct subclasses within astrocytic $(1 \mathrm{p} / 19 \mathrm{q}$-intact) LGGs [21]. One group, termed preglioblastoma (PG), consisted primarily of IDH-wild type tumors while the other two, termed neuroblastic (NB) and early progenitorlike (EPL), were almost exclusively IDH-mutant. These latter two subclasses were further delineated by different rates of TP53 mutation and their associations, by way of gene expression patterns, to distinct neuroglial precursor cell pools. Definitively linking these transcriptional subgroupings with specific pathogenic mutations would be of considerable interest.

To further explore the genomic landscape of LGG, particularly with reference to its different histopathological variants, we performed whole exome capture, nextgeneration sequencing on 4 WHO grade II gliomas followed by validation sequencing in an additional 28 . We found a strikingly high incidence of mutations in the ATRX gene ( $\alpha$ thalassemia/mental retardation syndrome $\mathrm{X}$-linked), entirely restricted to IDH-mutant LGGs of astrocytic lineage - astrocytomas and oligoastrocytomasand mutually exclusive with $1 \mathrm{p} / 19 \mathrm{q}$ codeletion. ATRX and its binding partner DAXX (death-associated protein 6) are central components of a chromatin remodeling complex required for the incorporation of $\mathrm{H} 3.3$ histone proteins into the telomeric regions of chromosomes [22, 23]. Dysfunction of the ATRX/DAXX complex results 
Table 2: Validated sequencing results for 32 LGGs showing mutations in IDH1, ATRX, and TP53. $1 \mathrm{p} / 19 \mathrm{q}$ status, astrocytoma transcriptional subclass [21], and ALT FISH results are also shown along with patient age, primary/recurrent tumor status, and pathological diagnosis. WT: wild type, A: astrocytoma, O: oligodendroglioma, OA: oligoastrocytoma, II: WHO grade II, III: WHO grade III, NB: neuroblastic, EPL: early progenitor-like, PG: preglioblastoma.

\begin{tabular}{|c|c|c|c|c|c|c|c|c|c|}
\hline Tumor & Age & \begin{tabular}{|l|} 
Primary/ \\
Recurrent \\
\end{tabular} & IDH1 & ATRX & TP53 & Pathology & $1 p / 19 q$ & \begin{tabular}{|l|} 
Astro \\
Subclass
\end{tabular} & \begin{tabular}{|l|} 
ALT \\
FISH \\
\end{tabular} \\
\hline G1 & 56 & Primary & p. R132H & WT & p.R141C & A II & intact & NB & NEG \\
\hline G2 & 27 & Recurrent & p. R132H & WT & WT & OA II & intact & NB & NEG \\
\hline G3 & 41 & Primary & p. R132H & WT & p.P90fs & OA II & intact & NB & POS \\
\hline G4 & 31 & Primary & p. R132H & WT & WT & A II & intact & NB & NEG \\
\hline G5 & 34 & Primary & p. R132G & p.K1001fs & p.R141C & A II & intact & EPL & POS \\
\hline G6 & 40 & Primary & p. R132H & p.L639fs & p.R141C & A II & intact & EPL & POS \\
\hline G7 & 35 & Primary & p. R132H & p.E991fs & p.G196A & A II & intact & EPL & POS \\
\hline G8 & 38 & Primary & p. R132H & p.R907X & p.R141C & OA II & intact & EPL & POS \\
\hline G9 & 41 & Primary & p. R132H & p.1338_1339del & WT & A II & intact & EPL & NEG \\
\hline G10 & 29 & Primary & p. R132C & p.1346_1347del & WT & OA II & intact & EPL & POS \\
\hline G11 & 58 & Recurrent & p. R132H & p.E1010fs & WT & OA II & intact & EPL & POS \\
\hline G12 & 38 & Recurrent & p. R132H & WT & p.L232R & A III & intact & EPL & NEG \\
\hline G13 & 43 & Recurrent & p. R132H & p.199_205del & p.R141C & A II & intact & EPL & POS \\
\hline G14 & 52 & Primary & p. R132H & p.K1018fs & p.R141C & A III & intact & EPL & POS \\
\hline G15 & 36 & Primary & p. R132H & p.R1302fs & p.101_103del & A III & intact & EPL & POS \\
\hline G16 & 34 & Primary & p. R132H & p.R221K & p.G196X & A III & intact & EPL & POS \\
\hline G17 & 36 & Primary & WT & WT & WT & A III & intact & EPL & NEG \\
\hline G18 & 32 & Primary & p. R132H & p.R1426X & WT & OA II & intact & $\mathrm{PG}$ & POS \\
\hline G19 & 62 & Primary & WT & WT & WT & A II & intact & $\mathrm{PG}$ & NEG \\
\hline G20 & 65 & Primary & WT & WT & WT & O III & intact & PG & NEG \\
\hline G21 & 34 & Recurrent & p.R132H & WT & WT & OA II & codeleted & N/A & NEG \\
\hline G22 & 41 & Recurrent & p.R132H & WT & WT & O II & codeleted & $\mathrm{N} / \mathrm{A}$ & N/A \\
\hline G23 & 38 & Primary & p.R132H & WT & WT & O II & codeleted & N/A & NEG \\
\hline G24 & 37 & Primary & p.R132H & WT & WT & O III & codeleted & N/A & NEG \\
\hline G25 & 52 & Recurrent & p.R132H & WT & WT & O II & codeleted & N/A & NEG \\
\hline G26 & 34 & Recurrent & p.R132H & WT & WT & O II & codeleted & N/A & NEG \\
\hline G27 & 45 & Primary & p.R132H & WT & WT & O II & codeleted & N/A & NEG \\
\hline G28 & 37 & Primary & p.R132H & WT & WT & O II & codeleted & $\mathrm{N} / \mathrm{A}$ & NEG \\
\hline G29 & 62 & Primary & p.R132H & WT & WT & O III & codeleted & N/A & NEG \\
\hline G30 & 46 & Primary & p.R132H & WT & WT & O III & codeleted & N/A & NEG \\
\hline G31 & 35 & Recurrent & p.R132H & WT & WT & O III & codeleted & N/A & NEG \\
\hline G32 & 58 & Primary & WT & WT & WT & O III & codeleted & N/A & NEG \\
\hline
\end{tabular}

in a phenomenon known as alternative lengthening of telomeres (ALT) along with more widespread genomic destabilization [24-26]. Mutations in ATRX and, to a lesser extent, $D A X X$ have recently been identified in a number of tumor subtypes, including pediatric and adult gliomas [24, 26-29]. In this report, we show that ATRX mutation is present in $\sim 70 \%$ of IDH-mutant, $1 \mathrm{p} / 19 \mathrm{q}$ intact
LGGs. Moreover, we demonstrate that ATRX mutation represents the defining molecular abnormality delineating the EPL subclass of LGG from other IDH-mutant astrocytic tumors. Finally, we correlate ATRX mutation with ALT, recapitulating a functional association with mitotic instability seen in other $A T R X$-mutant tumor types. 


\section{RESULTS}

\section{High-throughput resequencing demonstrates a high rate of ATRX mutation in IDH-mutant, 1p/19q-intact LGGs.}

To identify molecular abnormalities conspiring with IDH mutation in the pathogenesis of LGGs, we performed whole exome capture, next-generation sequencing on genomic DNA extracted from 4 WHO grade II gliomas - 3 astrocytomas and 1 oligoastrocytoma - with known mutations in IDH1. Additionally, all 4 tumors were $1 \mathrm{p} / 19 \mathrm{q}$-intact. DNA from case-matched blood was also sequenced to ascertain and screen out germline polymorphisms. We achieved a mean coverage of 57fold in targeted regions, with $80 \%$ of bases represented by at least 10 reads (Table S1). After quality filtering (see Material and Methods) our analysis revealed 376 candidate somatic mutations in 339 genes, which consisted of missense ( $85 \%$ ), nonsense (4\%), splice site $(5 \%)$, and insertion/deletion (4\%) variants (Table S2). We found that C:G to T:A transitions predominated, as has been reported for several other cancer subtypes [30] (Tables S3-S4).

We assembled promising candidate mutations based on coverage, mutational frequency, read quality, and predicted functional impact (see Materials and Methods for precise criteria) and subjected them to bi-directional Sanger sequencing validation. The resulting list of confirmed mutations demonstrated only 3 genes altered in more than one tumor: IDH1, TP53, and ATRX (Table 1). ATR $X$ mutation was present in 3 of 4 tumors, representing the most frequent genomic alteration in our sample set apart from IDH mutation.

To better determine the incidence of ATRX mutation across LGG subtypes, we performed high-throughput resequencing of all ATRX exons in 28 additional LGGs, along with case-matched blood. Our combined sample set consisted of 13 astrocytomas ( 8 WHO grade II and 5 WHO grade III), 12 oligodendrogliomas (6 WHO grade II and 6 WHO grade III), and 7 oligoastrocytomas (all WHO grade II). The coding regions of DAXX, TP53, $I D H 1$, and $I D H 2$ were also subjected to resequencing and results were correlated with known $1 \mathrm{p} / 19 \mathrm{q}$ status from de-identified patient records (Table 2). Once again, all candidate mutations were validated by Sanger sequencing. We found that $A T R X$ mutations were present in 12 tumors, all of which also harbored $I D H 1$ mutations. By contrast, $A T R X$ was uniformly wild type in the 4 IDH-wild type gliomas featured in our tumor cohort. DAXX mutations were not identified in either group. The pattern of ATRX mutations exhibited remarkable associations with other genomic abnormalities within IDH-mutant LGGs. For instance, $A T R X$ mutation was significantly correlated with TP53 mutation $(P=0.0189)$ and anticorrelated with $1 \mathrm{p} / 19 \mathrm{q}$ codeletion to the point of complete mutual exclusivity $(P=0.0003)$. All told, ATR $X$-mutant tumors accounted for $\sim 70 \%$ of IDH-mutant, 1p/19q-intact LGGs in our sample set. Given these findings, we were not surprised that $A T R X$ mutation was also strongly linked to astrocytic and oligoastrocytic morphology, as opposed to pure oligodendroglial morphology $(P=0.0009)$. No significant correlations were found between ATRX mutation and either patient age, primary/recurrent tumor status, or tumor grade. Similar to that seen previous reports $[24$, 26, 27], the distribution of ATRX mutations identified in our sample set was broad, spread across more than half of the protein, and consisted of frameshift (6), nonsense (2), missense (1), and in-frame deletion (3) variants. There were no readily apparent associations between the type or location of specific ATRX mutations and other genomic, demographic, or histopathological parameters, although our sample size likely precluded robust analysis in this regard.

\section{ATRX mutation defines the early progenitor-like molecular subclass of astrocytoma and correlates with ALT.}

As indicated above, our earlier work designated 3 molecularly and clinically distinct subclasses of astrocytic LGGs. To determine the composition of ATRX mutations within each subclass, we developed a multiplexed mRNA profiling assay on the Nanostring platform consisting of a 75 -gene signature ( 25 upregulated genes per subclass) and applied it to total RNA derived from $1 \mathrm{p} / 19 \mathrm{q}$-intact tumors in our sample set. Raw data was then processed through a classifying algorithm based on Linear Discriminant Analysis and trained on material from 24 astrocytic tumors of known subclass - 7 NB, 8 EPL, and 8 PG (FIG. 1A1B). Additionally, earlier work had already established the subclass assignment of 4 gliomas in our sequencing cohort [21].

We found a striking association between ATRX mutation and EPL subclass $(P=0.0044)$ (Table 2$)$. In total, $85 \%(11 / 13)$ of astrocytic LGGs designated as EPL by expression profiling harbored ATRX mutations, and $92 \%$ (11/12) of ATRX mutations were found in EPL tumors. Taken together, these findings indicate that ATRX mutation itself represents the defining molecular abnormality of a distinct astrocytoma subclass delineated by gene expression.

Previous studies have directly linked ATR $X$ mutation to ALT and genomic instability in a variety of tumors [24-26]. To assess whether ALT correlates with ATRX mutational status in LGG, we performed telomere FISH on our sample set. Consistent with earlier reports, we found a notably strong association between ATRX mutation and ALT $(P<0.0001)$, as indicated by ultrabright foci of intranuclear positivity by telomere FISH in multiple tumor 
cells (FIG. 2A-2B; Table 2). In total, 11/12 (92\% of LGGs with $A T R X$ mutations exhibited ALT, and 11/12 (92\%) of LGGs with ALT harbored ATRX mutations.

\section{DISCUSSION}

While the natural history of LGGs almost invariably culminates in their transformation to WHO grade IV tumors, their tendency to exhibit prolonged periods of indolent growth sharply contrasts with the biological behavior of GBMs [1]. This relative clinical stability likely reflects a less fundamentally altered physiological state, and provides an inviting "window of opportunity" for the implementation of appropriately targeted therapeutics. Elucidating the precise mechanisms driving LGG pathogenesis, therefore, is of vital importance to the advancement of their clinical management. The sheer frequency of IDH mutation in LGG demonstrates its biological relevance and highlights the unequivocal importance of epigenomics and metabolomics in gliomagenesis. Yet while its physiological effects are broad and profound, exactly how IDH mutation fundamentally drives a neoplastic phenotype remains unclear. Indeed, the inability of IDH mutation to promote glioma formation in mouse models thus far underscores this quandary, and further implies that additional molecular alterations are likely required for transformation.

We employed both whole exome and targeted next-generation sequencing approaches to identify

A

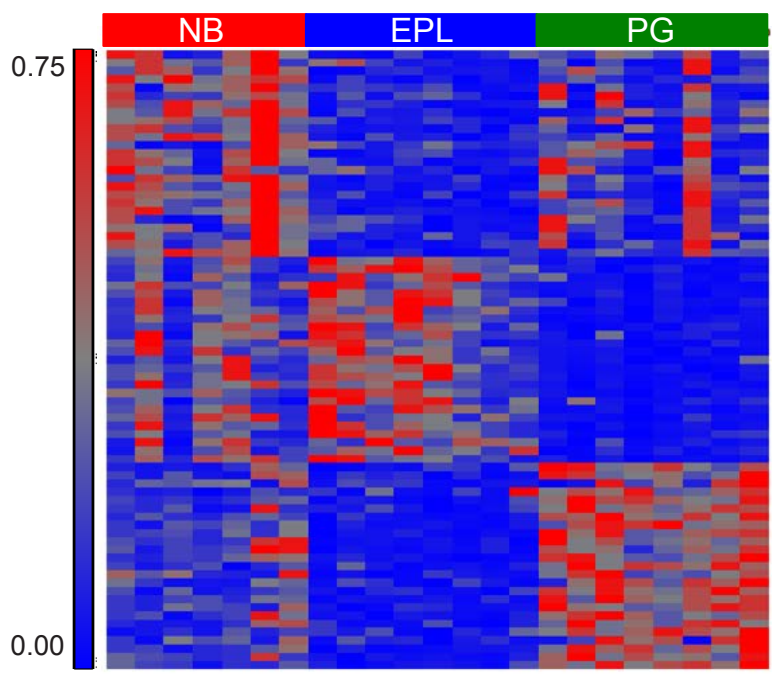

$A T R X$ mutations in a significant percentage of LGGs. Intriguingly, the distribution of $A T R X$ mutations tracked with specific diagnostic and molecularly defined tumor subclasses. Specifically, their presence was entirely restricted to IDH-mutant, 1p/19q-intact LGGs, astrocytic and oligoastrocytic in their morphology, where they were found in $\sim 70 \%$ of tumors and exhibited a tight correlation with TP53 mutation. These findings are strikingly consistent with those of a very recent study examining, among other parameters, ATRX, TP53, and IDH mutational status, along with $1 \mathrm{p} / 19 \mathrm{q}$ codeletion in a large cohort of low- and high-grade gliomas [28]. By contrast, a separate report, also very recent, found a somewhat lower rate of $A T R X$ abnormalities in astrocytic and oligoastrocytic LGGs $(\sim 42 \%)$ [29]. This discrepancy likely reflects the latter study's employment of immunohistochemical staining in tissue microarrays, and not sequencing-based mutational analysis, as its primary screening technique for ATRX abnormalities. Indeed, frequent ATRX mutations $(\sim 79 \%)$ were revealed in a smaller cohort of tumors actually subjected to sequencing in this same study. Regardless, together with ours, both reports support a fundamental molecular stratification of LGGs based on $I D H$ and $A T R X$ mutational status and $1 \mathrm{p} / 19 \mathrm{q}$ codeletion.

Additionally, our data indicate that abnormalities in $A T R X$ represent the defining molecular characteristic of the EPL subclass of astrocytic LGGs. We considered the possibility that functional loss of ATRX, a known chromatin regulator, might itself drive the EPL
B

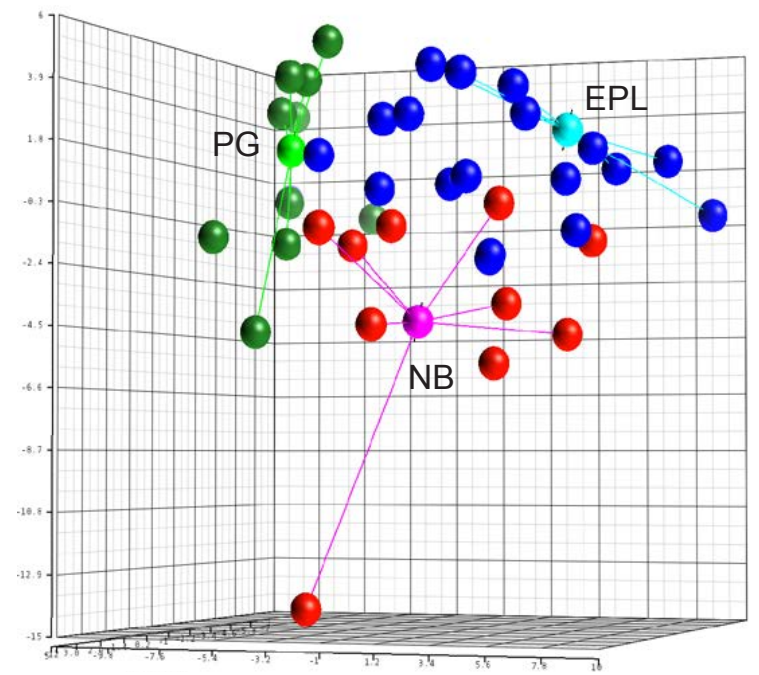

Figure 1: Nanostring classifier designates transcriptional subclasses of astrocytic LGG. A, standardized intensity plot showing 75-gene classifying signature applied to 23 training samples of known subclass. B, Nanostring profiling for training set and classified samples visualized by three dimensional principal component analysis. Centroids for training set samples are shown along with connectors. NB: neuroblastic, EPL: early progenitor-like, PG: preglioblastoma 
transcriptional signature. However, the recent finding that ATRX loss does not significantly alter H3.3 profiles across the coding genome argues against this [22], and suggests a more fundamental association between EPL subclass and specific cells of origin for IDH-mutant LGG. Our previous work established biological links between EPL tumors and early-stage neuroglial precursor cells in the mammalian subventricular zone (SVZ) [21]. The high frequency of $A T R X$ mutations in EPL tumors, therefore, implies either a unique propensity of this specific SVZ progenitor population to acquire $A T R X$ mutation or a particular sensitivity to its biological effects. Indeed, it has recently been suggested that regions harboring stemlike cells in the adult brain, including the SVZ, might be inherently more prone to oncogenic initiating events [31].

The invariable co-occurrence of $A T R X$ mutations with IDH mutations, and their frequent association with TP53 mutation support a cooperative pathogenic mechanism by which dysfunction in all three proteins is required for oncogenesis in a large subset of LGG. Recent work has shown that IDH mutation dramatically reprograms the cellular epigenome, the physiological effects of which include impaired differentiation and the abnormal maintenance of stem and progenitor cell populations in physiological states permissive to selfrenewal $[15,16,32]$. Additionally, multiple studies have demonstrated that loss of ATRX protein or ATRX mutation results in ALT and genomic instability [24-26, 28], and our own findings recapitulate these functional relationships in IDH-mutant LGGs. Combining genomic instability with inherent self-renewal potential could provide fertile ground for malignant transformation, particularly in the setting of TP53 loss, which would presumably allow affected cells to evade apoptosis and/or senescence [33]. More extensive in vitro and in vivo modeling in diseaserelevant experimental systems will be essential to test the validity of these and other related conjectures.

The recent identification of $\mathrm{H} 3.3$ histone protein mutations in pediatric GBM and diffuse intrinsic pontine glioma (DIPG) is particularly intriguing in light of our present findings $[26,34,35]$. Specifically, their frequent association with ATRX and TP53 mutations suggests a functional equivalence with IDH mutation in adult glioma, with both fundamentally altering global epigenomic landscape and cellular differentiation state. Similarly, the mutual exclusivity of $A T R X$ mutation with $1 \mathrm{p} / 19 \mathrm{q}$ deletion in adult IDH-mutant LGGs also implies analogous functionality, perhaps in the mediation of genomic instability. Thus, accruing evidence from direct molecular profiling in tumor tissue has bolstered the notion of a shared pathogenic mechanism, operative across a wide spectrum of glioma subtypes. While much work remains to be done, the detailed functional characterization of a common transformative pathway in LGG would have significant implications on future therapeutic development and disease management.

\section{METHODS}

\section{Glioma sample cohort}

Fresh frozen glioma tissue and de-identified demographic and clinical data was obtained from Memorial Sloan Kettering Cancer Center (MSKCC) Brain Tumor Center (BTC) following approval from the Institutional Review Board under the auspices of a blanket biospecimen utilization protocol. Frozen samples were mounted, sectioned, and stained with hematoxylin and eosin (H\& E) before being examined by a Neuropathologist (J.T.H.) for tumor adequacy. Specimens passing quality control were processed for genomic DNA and total RNA extraction using commercially available reagents (Qiagen, Valencia, CA, USA).

\section{High-throughput sequencing}

Whole exome capture of 4 LGG samples consisting of matched tumor/normal pairs was performed using the SureSelect 50MB Target Enrichment System (Agilent Technologies, Santa Clara, CA, USA) according to the manufacturer's standard protocol. Libraries were then subjected to $50 \mathrm{bp}+35 \mathrm{bp}$ paired-end sequencing on the SOLiD 5500xl platform (Applied Biosystems, Foster City,
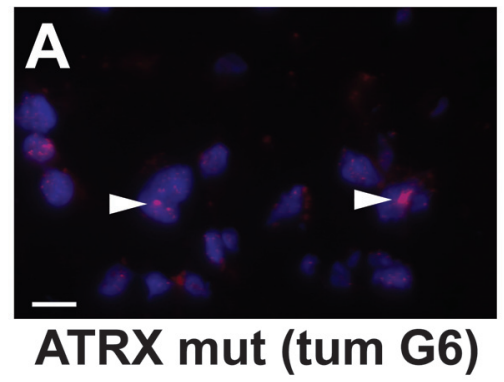

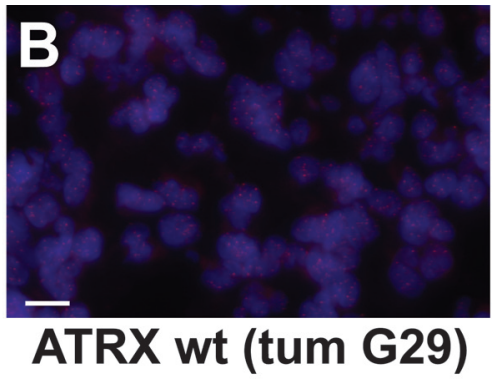

Figure 2: Telomere FISH demonstrates ALT in ATRX-mutant LGG. Representative micrographs showing telomere FISH (red) performed in ATRX-mutant (mut; A) and wild type (wt; B) LGGs. An intranuclear ALT-positive focus is indicated (arrowhead). Slides were counterstained with DAPI (blue). White scale bars are $120 \mu \mathrm{m}$. 
CA, USA). Sequencing reads were aligned to the reference human genome (hg19) using the BWA aligner (open source software). Somatic mutations were then identified using an automated computational pipeline (Mut(e)Piper). In its first stage, the pipeline consists of GATK [36] base quality score recalibration, indel realignment, and duplicate removal using standard parameters [37]. Mut(e) Piper then employs SomaticSniper [38] and Somatic Indel Detector (GATK) to identify single nucleotide variants (SNVs) and insertions/deletions. This process identified a total of 19883 somatic variants in our initial 4 sample pairs, ranging from 4329 to 6601 per tumor with an average of 4971 (Table S5). The data were filtered using cutoffs of 3\% allelic frequency for the variant allele in tumor and 97\% allelic frequency for the reference (hg19) allele in normal. The candidate variant list was then further pruned using 1000genomes, dbSNP132 and ESP5400 (NHLBI Exome Sequencing Project (ESP), Exome Variant Server) databases to exclude confirmed single nucleotide polymorphisms. Short-listed variants were annotated using ANNOVAR (UCSC Known Genes) [39] and somatic mutations were identified as non-synonymous substitutions and frameshift/non-frameshift deletions/ insertions. To assemble a list of high-value mutations for initial validation, we selected all recurrent mutations and non-recurrent mutations meeting the following criteria: 1) $20 X$ and $9 X$ coverage in normal and tumor samples respectively, 2) an average regional (100 bp) phred mapping quality score of $>15,3$ ) presence of mutations on nonduplicated reads, 4) mutational allelic frequency of $>15$, and 4) a MutationAssessor [40] functional impact score of $>1$. These filters produced a candidate list of 43 mutations involving 27 genes (Table S6) that were then subjected to Sanger Sequencing validation (see below). An expanded cohort of 28 additional tumors/normal pairs was subjected to focused resequencing for $A T R X, I D H 1 / 2$, $T P 53$, and $D A X X$ on the MiSeq platform (Illumina, San Diego, CA, USA). Exonic and intron-exon border regions for each gene were captured with a custom NimbleGen SeqCap EZ bait library for short reads (Roche, Madison, WI, USA), following the manufacturer's standard protocol. Mean coverage for captured regions ranged from 10 to 134 reads with an average of 86 (Table S7). Sequencing data was processed using the Mut(e)Piper pipeline (see above).

\section{Sanger sequencing}

Exons of interest (NCBI Human Genome Build 36.1) were broken into amplicons of $350 \mathrm{bp}$ or less, and specific primers were designed using Primer3 (http:// frodo.wi.mit.edu/primer3/) to cover exonic regions and $\sim 50$ bp of flanking intronic sequence. PCR reactions were carried out in 384 well plates, in a Duncan DT-24 water bath thermal cycler, with $10 \mathrm{ng}$ of template DNA (Repli-G Midi, Qiagen) using a touchdown PCR protocol with Kapa2G Fast HotStart Taq (Kapa Biosystems, Cape Town, South Africa) consisting of 1 cycle of $95^{\circ} \mathrm{C}$ for 5 min; 3 cycles of $95^{\circ} \mathrm{C}$ for $30 \mathrm{sec}, 64^{\circ} \mathrm{C}$ for $15 \mathrm{sec}, 72^{\circ} \mathrm{C}$ for $30 \mathrm{sec} ; 3$ cycles of $95^{\circ} \mathrm{C}$ for $30 \mathrm{sec}, 62^{\circ} \mathrm{C}$ for $15 \mathrm{sec}, 72^{\circ} \mathrm{C}$ for $30 \mathrm{sec} ; 3 \mathrm{cycles}$ of $95^{\circ} \mathrm{C}$ for $30 \mathrm{sec}, 60^{\circ} \mathrm{C}$ for $15 \mathrm{sec}$, $72^{\circ} \mathrm{C}$ for $30 \mathrm{sec} ; 37 \mathrm{cycles}$ of $95^{\circ} \mathrm{C}$ for $30 \mathrm{sec}, 58^{\circ} \mathrm{C}$ for 15 sec, $72^{\circ} \mathrm{C}$ for $30 \mathrm{sec} ; 1 \mathrm{cycle}$ of $70^{\circ} \mathrm{C}$ for $5 \mathrm{~min}$. Templates were purified using AMPure (Agencourt Biosciences, Beverly, MA, USA), split into two, and sequenced bidirectionally with M13 forward and reverse primers using the Big Dye Terminator Kit v.3.1 (Applied Biosystems) at Agencourt Biosciences. Dye terminators were removed using the CleanSEQ kit (Agencourt Biosciences), and sequence reactions were run on an ABI PRISM 3730xl sequencing apparatus (Applied Biosystems).

\section{Nanostring expression profiling}

We developed a Nanostring-based classifier for astrocytic LGGs based on previous work [21]. Briefly, Receiver Operator Characteristic (ROC) rankings for subclass-associated transcripts were used to identify 25 signature genes per subclass (Table S8). A specific CodeSet (Nanostring Technologies, Seattle, WA, USA) was derived for this gene list and applied, using a Nanostring nCounter, to RNA derived from 23 astrocytic LGGs of known subclass. Raw data was subjected to quantile normalization and used to train a Linear Discriminant Analysis classifying algorithm on the MultiExperiment Viewer (MeV) software platform (www.tm4. org/mev/). Nanostring data from our sequencing cohort was then processed through this pipeline for final tumor subclassification.

\section{ALT fluorescence in situ hybridization (FISH)}

$5 \mu \mathrm{m}$ frozen sections were fixed with $4 \%$ paraformaldehyde for 10 minutes, dehydrated, and denatured at $85{ }^{\circ} \mathrm{C}$ for 5 minutes in hybridization mixture consisting of $10 \mathrm{mM}$ Tris-HCL pH 7.2, 70\% formamide, $0.5 \%$ blocking solution reagent (Roche), and a complementary Cy3-labeled peptide nucleic acid probe specific to the mammalian telomere repeat sequence TTAGGGTTAGGGTTAGGG 3' (Applied Biosystems). Hybridization was performed at room temperature for two hours in the hybridization mixture. Slides were then washed twice with $70 \%$ formamide, $10 \mathrm{mM}$ Tris- $\mathrm{HCl} \mathrm{pH}$ 7.0-7.5, 0.1\% BSA followed by multiple washes in PBS. Finally, slides were mounted in Prolong Gold with DAPI (Invitrogen, Grand Island, NY, USA) and examined using a Leica CTR5000 inverted fluorescent microscope. ALT was identified by the presence of ultrabright, clumpy, intranuclear foci as previously described [24]. Companion $\mathrm{H}$ $\&$ E stained sections were examined in all cases to localize tumor tissue. 


\section{Statistics}

Univariate associations were evaluated using two-tailed Fisher's exact tests. Stated $P$ values do not reflect Bonferroni correction. Please see other Materials and Methods subsections for statistical considerations involving specific analytical pipelines.

\section{ACKOWLEDGEMENTS}

We would like to thank Juan Li, Janice Cheng, Maryam Hassimi, Jeffrey Zhou, and Agnes Viale in the MSKCC Genomics Core Lab for the technical support. Similarly, we would like to acknowledge Olga Aminova, Igor Dogalev, Sabrina Thomas, and Andrew Kayserian in the Geoffrey Beene Translational Oncology Core Facility for their assistance. Finally, we would like to thank Cameron W. Brennan, Marc K. Rosenblum, and Eric C. Holland for their helpful comments and suggestions. This work was supported by the MSKCC Brain Tumor Center, the Leon Levy Foundation, the Geoffrey Beene Foundation, the Sidney Kimmel Foundation, the Doris Duke Charitable Foundation, and the AACR/Landon Foundation.

\section{Conflicts of Interest}

The authors declare no conflicts of interest.

\section{REFERENCE}

1. Wen PY and Kesari S. Malignant Gliomas in Adults. The New England journal of medicine. 2008; 359:492-507.

2. Noushmehr H, Weisenberger DJ, Diefes K, Phillips HS, Pujara K, Berman BP, Pan F, Pelloski CE, Sulman EP, Bhat KP, Verhaak RG, Hoadley KA, Hayes DN, Perou CM, Schmidt HK, Ding L, et al. Identification of a CpG island methylator phenotype that defines a distinct subgroup of glioma. Cancer Cell. 2010; 17(5):510-522.

3. Parsons DW, Jones S, Zhang X, Lin JC-H, Leary RJ, Angenendt P, Mankoo P, Carter H, Siu I-M, Gallia GL, Olivi A, McLendon R, Rasheed BA, Keir S, Nikolskaya T, Nikolsky Y, et al. An Integrated Genomic Analysis of Human Glioblastoma Multiforme. Science (New York, NY. 2008; 321:1807-1812.

4. The Cancer Genome Atlas Research Network. Comprehensive genomic characterization defines human glioblastoma genes and core pathways. Nature. 2008; 455:1061-1068.

5. Verhaak RGW, Hoadley KA, Purdom E, Wang V, Qi Y, Wilkerson MD, Miller CR, Ding L, Golub TR, Mesirov JP, Alexe G, Lawrence M, O'Kelly M, Tamayo P, Weir BA, Gabriel S, et al. An integrated genomic analysis identifies clinically relevant subtypes of glioblastoma characterized by abnormalities in PDGFRA, IDH1, EGFR, and NF1. Cancer Cell. 2009; 17:98-110.

6. Parsons DW. The evolving picture of the glioblastoma genome. Oncotarget. 2010; 1(4):237-238.

7. Duncan CG, Killela PJ, Payne CA, Lampson B, Chen WC, Liu J, Solomon D, Waldman T, Towers AJ, Gregory SG, McDonald KL, McLendon RE, Bigner DD and Yan H. Integrated genomic analyses identify ERRFI1 and TACC3 as glioblastoma-targeted genes. Oncotarget. 2010; 1(4):265277.

8. Weber GL, Parat MO, Binder ZA, Gallia GL and Riggins GJ. Abrogation of PIK3CA or PIK3R1 reduces proliferation, migration, and invasion in glioblastoma multiforme cells. Oncotarget. 2011; 2(11):833-849.

9. Louis DN, Ohgaki H, Wiestler OD, Cavenee WK, Burger PC, Jouvet A, Scheithauer BW and Kleihues P. The 2007 WHO classification of tumours of the central nervous system. Acta neuropathologica. 2007; 114(2):97-109.

10. Sanson M, Marie Y, Paris S, Idbaih A, Laffaire J, Ducray F, El Hallani S, Boisselier B, Mokhtari K, Hoang-Xuan $\mathrm{K}$ and Delattre JY. Isocitrate dehydrogenase 1 codon 132 mutation is an important prognostic biomarker in gliomas. J Clin Oncol. 2009; 27(25):4150-4154.

11. Yan H, Parsons DW, Jin G, McLendon R, Rasheed BA, Yuan W, Kos I, Batinic-Haberle I, Jones S, Riggins GJ, Friedman H, Friedman A, Reardon D, Herndon J, Kinzler $\mathrm{KW}$, Velculescu VE, et al. IDH1 and IDH2 mutations in gliomas. The New England journal of medicine. 2009; 360(8):765-773.

12. Hartmann C, Meyer J, Balss J, Capper D, Mueller W, Christians A, Felsberg J, Wolter M, Mawrin C, Wick W, Weller M, Herold-Mende C, Unterberg A, Jeuken JW, Wesseling P, Reifenberger G, et al. Type and frequency of IDH1 and IDH2 mutations are related to astrocytic and oligodendroglial differentiation and age: a study of 1,010 diffuse gliomas. Acta neuropathologica. 2009; 118(4):469474.

13. Dang L, White DW, Gross S, Bennett BD, Bittinger MA, Driggers EM, Fantin VR, Jang HG, Jin S, Keenan MC, Marks KM, Prins RM, Ward PS, Yen KE, Liau LM, Rabinowitz JD, et al. Cancer-associated IDH1 mutations produce 2-hydroxyglutarate. Nature. 2009; 462(7274):739744.

14. Ward PS, Patel J, Wise DR, Abdel-Wahab O, Bennett BD, Coller HA, Cross JR, Fantin VR, Hedvat CV, Perl AE, Rabinowitz JD, Carroll M, Su SM, Sharp KA, Levine RL and Thompson CB. The common feature of leukemiaassociated IDH1 and IDH2 mutations is a neomorphic enzyme activity converting alpha-ketoglutarate to 2-hydroxyglutarate. Cancer Cell. 2010; 17(3):225-234.

15. Lu C, Ward PS, Kapoor GS, Rohle D, Turcan S, AbdelWahab O, Edwards CR, Khanin R, Figueroa ME, Melnick A, Wellen KE, O'Rourke DM, Berger SL, Chan TA, Levine RL, Mellinghoff IK, et al. IDH mutation impairs histone demethylation and results in a block to cell differentiation. 
Nature. 2012; 483(7390):474-478.

16. Turcan S, Rohle D, Goenka A, Walsh LA, Fang F, Yilmaz E, Campos C, Fabius AW, Lu C, Ward PS, Thompson CB, Kaufman A, Guryanova O, Levine R, Heguy A, Viale A, et al. IDH1 mutation is sufficient to establish the glioma hypermethylator phenotype. Nature. 2012; 483(7390):479483.

17. Cairncross JG, Ueki K, Zlatescu MC, Lisle DK, Finkelstein DM, R.R. H, Silver JS, Stark PC, MacDonald DR, Ino Y, Ramsay DA and Louis DN. Specific genetic predictors of chemotherapeutic response and survival in patients with anaplastic oligodendrogliomas. J Natl Cancer Inst. 1998; 90:1473-1479.

18. Ducray F, El Hallani S and Idbaih A. Diagnostic and prognostic markers in gliomas. Current opinion in oncology. 2009.

19. Reifenberger G, Reifenberger J, Liu L, James CD, Wechsler W and Collins VP. Molecular genetic analysis of oligodendroglial tumors shows preferential allelic deletions on 19q and 1p. Am J Pathol. 1994; 145:1175-1190.

20. Thon N, Eigenbrod S, Kreth S, Lutz J, Tonn JC, Kretzschmar H, Peraud A and Kreth FW. IDH1 mutations in grade II astrocytomas are associated with unfavorable progression-free survival and prolonged postrecurrence survival. Cancer. 2011; 118(2):452-460.

21. Gorovets D, Kannan K, Shen R, Kastenhuber ER, Islamdoust N, Campos C, Pentsova E, Heguy A, Jhanwar SC, Mellinghoff IK, Chan TA and Huse JT. IDH mutation and neuroglial developmental features define clinically distinct subclasses of lower grade diffuse astrocytic glioma. Clin Cancer Res. 2012; 18(9):2490-2501.

22. Goldberg AD, Banaszynski LA, Noh KM, Lewis PW, Elsaesser SJ, Stadler S, Dewell S, Law M, Guo X, Li X, Wen D, Chapgier A, DeKelver RC, Miller JC, Lee YL, Boydston EA, et al. Distinct factors control histone variant H3.3 localization at specific genomic regions. Cell. 2010; 140(5):678-691.

23. Lewis PW, Elsaesser SJ, Noh KM, Stadler SC and Allis CD. Daxx is an H3.3-specific histone chaperone and cooperates with ATRX in replication-independent chromatin assembly at telomeres. Proc Natl Acad Sci U S A. 2010; 107(32):14075-14080.

24. Heaphy CM, de Wilde RF, Jiao Y, Klein AP, Edil BH, Shi C, Bettegowda C, Rodriguez FJ, Eberhart CG, Hebbar S, Offerhaus GJ, McLendon R, Rasheed BA, He Y, Yan H, Bigner DD, et al. Altered telomeres in tumors with ATRX and DAXX mutations. Science (New York, NY. 2011; 333(6041):425.

25. Lovejoy CA, Li W, Reisenweber S, Thongthip S, Bruno J, de Lange T, De S, Petrini JH, Sung PA, Jasin M, Rosenbluh J, Zwang Y, Weir BA, Hatton C, Ivanova E, Macconaill L, et al. Loss of ATRX, Genome Instability, and an Altered DNA Damage Response Are Hallmarks of the Alternative Lengthening of Telomeres Pathway. PLoS Genet. 2012; 8(7):e1002772.
26. Schwartzentruber J, Korshunov A, Liu XY, Jones DT, Pfaff E, Jacob K, Sturm D, Fontebasso AM, Quang DA, Tonjes M, Hovestadt V, Albrecht S, Kool M, Nantel A, Konermann C, Lindroth A, et al. Driver mutations in histone H3.3 and chromatin remodelling genes in paediatric glioblastoma. Nature. 2012; 482(7384):226-231.

27. Jiao Y, Shi C, Edil BH, de Wilde RF, Klimstra DS, Maitra A, Schulick RD, Tang LH, Wolfgang CL, Choti MA, Velculescu VE, Diaz LA, Jr., Vogelstein B, Kinzler KW, Hruban RH and Papadopoulos N. DAXX/ATRX, MEN1, and mTOR pathway genes are frequently altered in pancreatic neuroendocrine tumors. Science (New York, NY. 2011; 331(6021):1199-1203.

28. Jiao Y, Killela PJ, Reitman ZJ, Rasheed AB, Heaphy CM, de Wilde RF, Rodriguez FJ, Rosemberg S, Oba-Shinjo SM, Marie SK, Bettegowda C, Agrawal N, Lipp E, Pirozzi C, Lopez G, He Y, et al. Frequent ATRX, CIC, and FUBP1 mutations refine the classification of malignant gliomas. Oncotarget. 2012.

29. Liu XY, Gerges N, Korshunov A, Sabha N, KhuongQuang DA, Fontebasso AM, Fleming A, Hadjadj D, Schwartzentruber J, Majewski J, Dong Z, Siegel P, Albrecht S, Croul S, Jones DT, Kool M, et al. Frequent ATRX mutations and loss of expression in adult diffuse astrocytic tumors carrying IDH1/IDH2 and TP53 mutations. Acta neuropathologica. 2012.

30. Greenman C, Stephens P, Smith R, Dalgliesh GL, Hunter C, Bignell G, Davies H, Teague J, Butler A, Stevens C, Edkins S, O’Meara S, Vastrik I, Schmidt EE, Avis T, Barthorpe $\mathrm{S}$, et al. Patterns of somatic mutation in human cancer genomes. Nature. 2007; 446(7132):153-158.

31. Munoz DM and Guha A. Mouse models to interrogate the implications of the differentiation status in the ontogeny of gliomas. Oncotarget. 2011; 2(8):590-598.

32. Figueroa ME, Abdel-Wahab O, Lu C, Ward PS, Patel J, Shih A, Li Y, Bhagwat N, Vasanthakumar A, Fernandez HF, Tallman MS, Sun Z, Wolniak K, Peeters JK, Liu W, Choe SE, et al. Leukemic IDH1 and IDH2 mutations result in a hypermethylation phenotype, disrupt TET2 function, and impair hematopoietic differentiation. Cancer Cell. 2010; 18(6):553-567.

33. Negrini S, Gorgoulis VG and Halazonetis TD. Genomic instability--an evolving hallmark of cancer. Nat Rev Mol Cell Biol. 2010; 11(3):220-228.

34. Khuong-Quang DA, Buczkowicz P, Rakopoulos P, Liu XY, Fontebasso AM, Bouffet E, Bartels U, Albrecht S, Schwartzentruber J, Letourneau L, Bourgey M, Bourque G, Montpetit A, Bourret G, Lepage P, Fleming A, et al. K27M mutation in histone $\mathrm{H} 3.3$ defines clinically and biologically distinct subgroups of pediatric diffuse intrinsic pontine gliomas. Acta neuropathologica. 2012.

35. Wu G, Broniscer A, McEachron TA, Lu C, Paugh BS, Becksfort J, Qu C, Ding L, Huether R, Parker M, Zhang J, Gajjar A, Dyer MA, Mullighan CG, Gilbertson RJ, Mardis $\mathrm{ER}$, et al. Somatic histone $\mathrm{H} 3$ alterations in pediatric diffuse 
intrinsic pontine gliomas and non-brainstem glioblastomas. Nat Genet. 2012; 44(3):251-253.

36. McKenna A, Hanna M, Banks E, Sivachenko A, Cibulskis K, Kernytsky A, Garimella K, Altshuler D, Gabriel S, Daly $\mathrm{M}$ and DePristo MA. The Genome Analysis Toolkit: a MapReduce framework for analyzing next-generation DNA sequencing data. Genome Res. 2010; 20(9):1297-1303.

37. DePristo MA, Banks E, Poplin R, Garimella KV, Maguire JR, Hartl C, Philippakis AA, del Angel G, Rivas MA, Hanna M, McKenna A, Fennell TJ, Kernytsky AM, Sivachenko AY, Cibulskis K, Gabriel SB, et al. A framework for variation discovery and genotyping using next-generation DNA sequencing data. Nat Genet. 2011; 43(5):491-498.

38. Larson DE, Harris CC, Chen K, Koboldt DC, Abbott TE, Dooling DJ, Ley TJ, Mardis ER, Wilson RK and Ding L. SomaticSniper: identification of somatic point mutations in whole genome sequencing data. Bioinformatics. 2012; 28(3):311-317.

39. Wang K, Li M and Hakonarson H. ANNOVAR: functional annotation of genetic variants from high-throughput sequencing data. Nucleic Acids Res. 2010; 38(16):e164.

40. Reva B, Antipin Y and Sander C. Predicting the functional impact of protein mutations: application to cancer genomics. Nucleic Acids Res. 2011; 39(17):e118. 